\title{
Pericyte adhesion is impaired on extracellular matrix produced by endothelial cells in high hexose concentrations
}

\author{
E. Beltramo, F. Pomero, A. Allione, F. D’Alù, E. Ponte, M. Porta \\ Department of Internal Medicine, University of Turin, Torino, Italy
}

\begin{abstract}
Aims/hypothesis. Thickening of the basement membrane and selective loss of pericytes are early events in diabetic retinopathy. We aimed at checking whether pericyte interaction with extracellular matrix produced by endothelial cells is influenced by the hexose concentrations in which endothelial cells are cultured. Methods. Conditioned extracellular matrixes were obtained by growing human umbilical vein endothelial cells in media containing $28 \mathrm{mmol} / \mathrm{l}$ hexoses (Dglucose, D-galactose, L-glucose), which undergo different intracellular processing, before and after adding the inhibitors of protein glycation thiamine or aminoguanidine. Having removed the endothelium, bovine retinal pericytes were grown on such matrixes and, in separate experiments, on laminin, fibronectin or type IV collagen. Pericyte adhesion was determined by cell counts $18 \mathrm{~h}$ after seeding.
\end{abstract}

Results. Reduced adhesion was observed on matrixes produced in high D-glucose, high D-galactose and high L-glucose. Both thiamine and aminoguanidine restored impaired pericyte adhesion when added to high D-glucose and high D-galactose, but not L-glucose. Laminin, fibronectin and type IV collagen did not consistently modify pericyte adhesion.

Conclusions/interpretations. Pericyte adhesion is impaired on extracellular matrix produced by endothelium in high hexose concentrations. This could result from excess protein glycation, corrected by aminoguanidine and thiamine, rather than altered glycoprotein composition. [Diabetologia (2002) 45: 416419]

Keywords Diabetic retinopathy, extracellular matrix, pericytes, endothelium, glucose, galactose, aminoguanidine, thiamine, glycation.
Thickening of the basement membrane occurs early on in the natural history of diabetic microangiopathy [1]. Qualitative and quantitative changes of the extracellular matrix (ECM) making up the capillary basement membrane could play a major role in the subsequent evolution of diabetic retinopathy. Although the

\footnotetext{
Received: 28 June 2001 and in revised form: 16 November 2001
}

Corresponding author: Dr. E. Beltramo, Department of Internal Medicine, University of Turin, C.so AM Dogliotti, 14, Torino, Italy, e-mail: elena.beltramo@unito.it

Abbreviations: ECM, Extracellular matrix; M199, medium 199 HEPES modification; BRP, bovine retinal pericytes; ECGS, endothelial cell growth supplement; AG, aminoguanidine; HUVEC, human umbilical vein endothelial cells mechanism(s) are still not clear, some ECM components influence the behaviour of endothelial cells and the characteristics of ECM-associated growth factors [2].

Endothelium probably is the major contributor to the synthesis of ECM material. Endothelial cells increase their synthesis of type IV collagen and fibronectin in particular when cultured in high glucose concentrations [2,3]. ECM produced in high glucose has been reported to reduce endothelial cell replication, even in the absence of high ambient glucose [2].

However, loss of endothelial cells is a later event of diabetic retinopathy, whereas selective loss of pericytes, which are embedded within the ECM, is thought to occur earlier [1], possibly along with basement membrane thickening. 


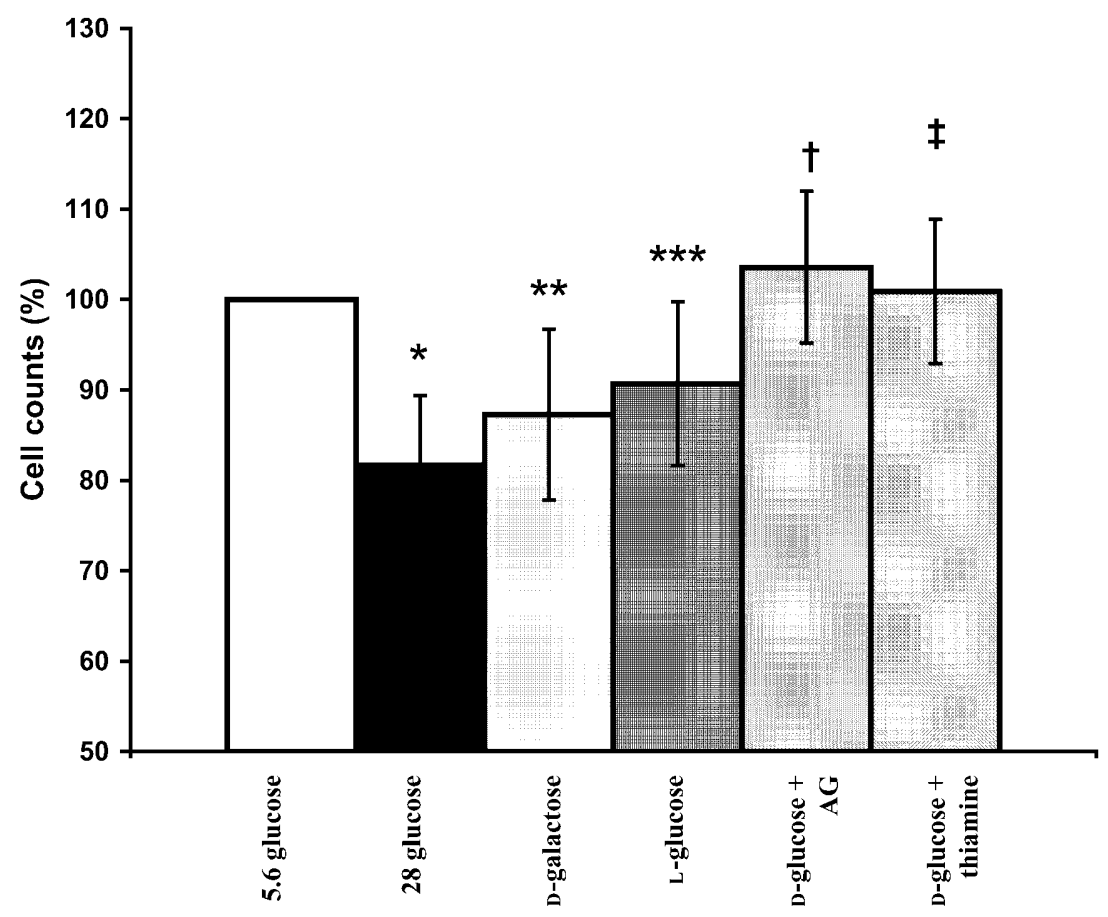

Fig. 1. Adhesion (counts) of BRP grown in normal glucose on ECM produced in six different conditions, expressed as percentages of positive control. $* p=0.000$ vs ECM in normal glucose, $* * * * * p=0.004$ vs ECM in normal glucose, $* * * p=0.015$ vs $\mathrm{ECM}$ in normal glucose, ${ }^{\dagger} p=0.000$ vs high D-glucose, $\$ p=0.002$ vs high D-glucose

This study investigated if the production of abnormal ECM by endothelial cells cultured in high hexose concentrations could influence pericyte adhesion.

\section{Materials and methods}

Cell cultures. Human umbilical vein endothelial cells (HUVECs) were obtained from human umbilical cords as previously described [4]. Pools of cells from 3 to 5 cords were grown in Medium 199 - HEPES Modification (M199), added with $20 \%$ FCS, until confluent. In secondary cultures, HUVECs were kept in M199+20\% FCS $+50 \mu \mathrm{g} / \mathrm{ml}$ endothelial cell growth supplement (ECGS). All reagents were purchased from Sigma Chemical, St. Louis, Mo., USA.

Bovine retinal pericytes (BRP) were obtained from pools of 15-20 bovine retinas as previously described [5]. BRP were characterized by $3 \mathrm{G} 5$ (a specific membrane ganglioside) fluorescence immunostaining [5]. They were grown in DMEM $5.6 \mathrm{mmol} / 1$ glucose with $10 \% \mathrm{FCS}$.

ECM production by HUVEC. HUVECs in primary culture were detached by trypsin-EDTA and seeded in 6 -well plates (about $1 \times 10^{5}$ cells/well, 12500 cells $/ \mathrm{cm}^{2}$ ) with $2 \mathrm{ml}$ medium. After $24 \mathrm{~h}$, the medium was removed and a media containing 5.6 or $28 \mathrm{mmol} / 1$ D-glucose, $5.6 \mathrm{mmol} / \mathrm{l}$ D-glucose and $22.4 \mathrm{mmol} / \mathrm{l} \mathrm{D}$-galactose, $5.6 \mathrm{mmol} / \mathrm{l} \mathrm{D}$-glucose and $22.4 \mathrm{mmol} / \mathrm{l}$ L-glucose, with or without $7 \mathrm{mmol} / \mathrm{l}$ aminoguanidine $(\mathrm{AG})$ or $150 \mu \mathrm{mol} / 1$ thiamine were added. After 7 days in culture, the wells were washed with PBS and treated for $4 \mathrm{~min}$ with
$0.2 \mathrm{~mol} / \mathrm{l} \mathrm{NH} \mathrm{NH}_{4} \mathrm{OH}$ to detach cells without affecting the matrix. The wells were then washed 4 times with PBS.

$B R P$ adhesion. BRP in primary culture were trypsinized and seeded in 6 -well plates (about $1 \times 10^{5}$ cells/well) on HUVECconditioned ECM. They were kept in physiological glucose for $18 \mathrm{~h}$ to allow enough time for these cells, which have $62 \mathrm{~h}$ duplication time [6], to attach but not to start dividing. They were then counted in Bürker chambers, after Trypan blue $0.2 \%$ colouring.

Experiments with ECM glycoproteins. Six-well plates were coated overnight at $37^{\circ} \mathrm{C}$ with either laminin $(0,10,25 \mu \mathrm{g} / \mathrm{ml}$ in PBS), fibronectin $(0,10,50 \mu \mathrm{g} / \mathrm{ml}$ in PBS) or type IV collagen $(0,10,50 \mu \mathrm{g} / \mathrm{ml}$ in PBS). Plates were then washed with PBS and BRP cultured for $18 \mathrm{~h}$ in medium with 5.6 glucose.

Statistical analysis. Because of the batch-to-batch variations, data are expressed as percentages (means \pm SD) of the results obtained with positive control conditions (ECM obtained from HUVEC in $5.6 \mathrm{mmol} / \mathrm{l}$ glucose, or plastic in the case of experiments with ECM glycoproteins) within each experiment. Statistical comparison among groups was carried out by one-way analysis of variance and two-tailed Student's $t$ test for paired data. Results were considered to be statistically significant if the $p$ value was 0.05 or less.

\section{Results}

BRP adhesion on HUVEC-conditioned ECM. BRP adhesion $(n=7)$ on ECM produced by HUVEC in $5.6 \mathrm{mmol} / \mathrm{l}$ glucose was higher than on plastic alone $(139.0 \pm 13.1 \%, p=0.010)$. BRP adhesion on ECM produced in high glucose was lower than on plastic $(86.9 \pm 13.3 \%, p=0.033)$ and ECM produced in normal glucose, $p=0.001$.

BRP adhesion on ECM obtained in high glucose $(n=9)$ was $81.9 \pm 7.5 \%$ of the positive control in nor- 


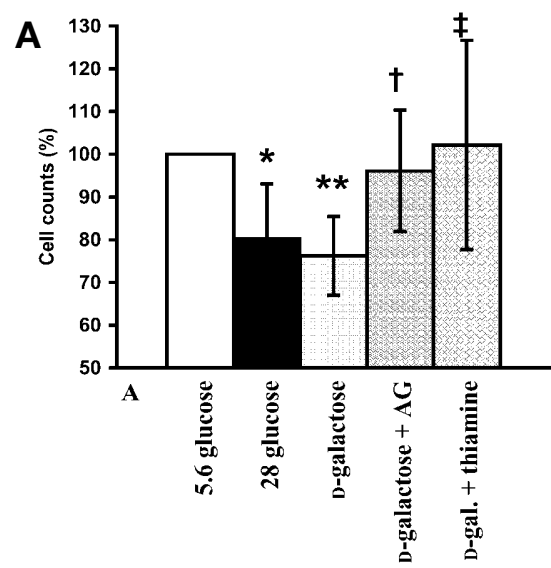

Fig. 2 (A, B). A Adhesion (counts) of BRP grown in normal glucose on ECM produced in D-galactose, with or without AG and thiamine, expressed as percentages of positive control. $* p=0.006$ vs ECM in normal glucose, $* * p=0.000$ vs ECM in normal glucose, ${ }^{\dagger} p=0.013$ vs high D-galactose, $\ddagger p=0.047$ vs high D-galactose. B Adhesion (counts) of BRP grown in normal glucose on ECM produced in L-glucose, with or without AG and thiamine, expressed as percentages of positive control. $* p=0.001$ vs ECM in normal glucose, $* * p=0.016$ vs ECM in normal glucose

mal glucose $(p=0.000)$. Adhesion on ECM obtained in high D-galactose was $87.3 \pm 9.5 \%$ of control $(p=0.004), 90.7 \pm 9.1 \%(p=0.015)$ in high L-glucose, $103.6 \pm 8.4 \%$ ( $p=$ NS vs normal glucose, $p=0.000$ vs high D-glucose) in high D-glucose $+A G$ and $100.9 \pm 8.0 \% \quad(p=\mathrm{NS} \quad$ vs normal glucose and $p=0.002$ vs high D-glucose) in high D-glucose + thiamine (Fig. 1).

In the following step, we measured BRP adhesion on ECM obtained adding AG or thiamine to high Dgalactose or L-glucose. The results were $(n=7$ in both cases): ECM produced in high $\mathrm{D}$-galactose $76.2 \pm 9.3 \%$ of control in 5.6 glucose $(p=0.000)$, high D-galactose + AG $96.1 \pm 14.2 \%$ ( $p=$ NS vs normal glucose, $p=0.013$ vs high D-galactose), and high D-galactose + thiamine $102.2 \pm 24.5 \%$ ( $p=$ NS vs normal glucose, $p=0.047 \mathrm{vs} \mathrm{high} \mathrm{D-galactose)}$. Counts on ECM obtained in high D-glucose were $80.5 \pm 12.6 \%$ of control ( $p=0.006)$ (Fig. 2A).

Counts on ECM produced in high L-glucose were $85.4 \pm 11.6 \%$ of control in $5.6 \mathrm{mmol} / \mathrm{l} \mathrm{D}$-glucose $(p=0.016), \quad$ high $\quad$ L-glucose $+\mathrm{AG} \quad 89.3 \pm 20.7 \%$ ( $p=$ NS vs both normal glucose and high L-glucose) and high L-glucose + thiamine $95.5 \pm 15.3 \%(p=\mathrm{NS}$ vs both normal glucose and high L-glucose). Counts on ECM obtained in high D-glucose were $71.2 \pm 13.8 \%(p=0.001)$ (Fig. 2 B $)$.

BRP adhesion on plates coated with ECM glycoproteins. No consistent changes were found in cell counts of BRP seeded in culture wells pre-treated with lami-

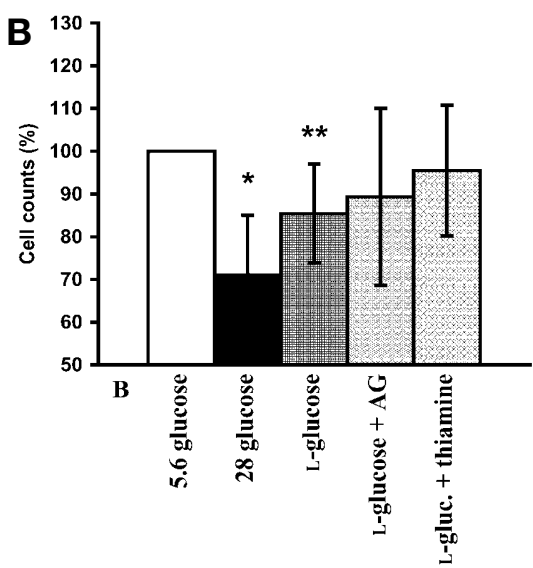

nin, fibronectin and type IV collagen (data not shown).

\section{Discussion}

ECMs produced by HUVECs in the presence of high D-glucose, D-galactose and L-glucose affected BRP adhesion to a similar extent, and addition of aminoguanidine and thiamine to high D-glucose and D-galactose normalised their counts. Metabolic or non enzymatic mechanism(s) or both could be involved in the generation of abnormal ECM material. All three hexoses tested are able to directly induce non-enzymatic glycosilation of intracellular and extracellular proteins [3], whereas only D-glucose and, possibly, Dgalactose could do so indirectly, through their metabolites. D-galactose is partially metabolised by the cell and could be transformed into D-glucose through the UDP-hexose pathway involved in the synthesis of glycogen and glycoproteins; L-glucose is internalised but not metabolised.

Glucose-6-phosphate, fructose-6-phosphate, glyceraldehyde-3-phosphate, and a polyol pathway-related metabolite, fructose-3-phosphate, along with its breakdown product, 3-deoxyglucosone, are extremely potent glycating agents, able to generate AGEs within $24 \mathrm{~h}$, whereas D-glucose and D-galactose need much longer incubation times [7]. Aminoguanidine inhibits the stabilisation of labile adducts into AGEs [6] and thiamine could prevent the generation of active glycating metabolites in high ambient D-glucose by shifting glyceraldehyde-3-phosphate towards the pentose phosphate cycle and, through activation of $\alpha$-keto-glutarate-dehydrogenase, by facilitating the utilization in the Krebs cycle of acetyl-CoA derived from accelerated glycolysis $[4,8]$. The relatively short exposure of HUVECs to high hexoses and aminoguanidine or thiamine in this work (7 days) suggests that both agents inhibit initial rather than late stages of glycation. In particular, the observation that thiamine and aminoguanidine normalised BRP adhesion to matrix obtained in high $\mathrm{D}$-glucose and D-galactose 
but not high L-glucose suggests that excess glycation resulting from overproduction of intermediate metabolites was involved in this phenomenon. This interpretation is consistent with previous reports that the key intermediate of AGE formation, methylglyoxal, interferes with crucial cell-matrix interactions by reacting with the arginine residues of collagen, thus inhibiting adhesion and subsequent spreading of two cell lines, MG63 and HT1080 [9]. The results with L-glucose, however, suggest that a minor role for direct glycation or osmotic mechanisms or both cannot be ruled out.

BRP adhered more to ECM obtained in normal glucose than plastic alone, in accordance with previous reports [6]. ECM obtained in high D-glucose was less viable than plastic. Abnormal expression of adhesion molecules on these ECMs could be involved in this phenomenon.

High glucose increased type IV collagen and fibronectin mRNA synthesis and upregulated transcription of their genes in cultured human endothelial cells [3]. Increased synthesis of basement membrane collagen in the retina has been found in diabetic rats [10], while fibronectin immunostaining increases in the retina of diabetic patients with background retinopathy [2]. To check whether these glycoproteins modified pericyte adhesion, and therefore whether their presence in abnormal amounts in HUVEC-conditioned ECMs could contribute to the results described above, BRP were cultured on surfaces which had been pre-treated with fibronectin, laminin and type IV collagen. Because no consistent changes were observed in BRP adhesion with increasing concentrations of any of these glycoproteins, it is not likely that these - if produced in excess by HUVEC in high glucose - played a role in our system.
Acknowledgements. This work was supported by grants from the University of Turin (Fondi ex-60\%) and the Italian Ministry of Health. We gratefully acknowledge the help of D. Ruggiero and N. Wiernsperger, of Lypha-Inserm, Villeurbanne, France, for fruitful suggestions and discussion.

\section{References}

1. Siperstein MD (1988) Diabetic microangiopathy, genetics, enviroment, and treatment. Am J Med 85 [Suppl 5A]: 119-130

2. Podestà F, Roth T, Ferrara F, Cagliero E, Lorenzi M (1997) Cytoskeletal changes induced by excess extracellular matrix impair endothelial cell replication. Diabetologia 40: 879-886

3. Cagliero E, Roth T, Roy S, Lorenzi M (1991) Characteristics and mechanisms of high-glucose-induced overexpression of basement membrane components in cultured human endothelial cells. Diabetes 40: 102-110

4. La Selva M, Beltramo E, Pagnozzi F et al. (1996) Thiamine corrects delayed replication and decreases production of lactate and advanced glycation end-products in bovine retinal and human umbilical vein endothelial cells cultured under high glucose conditions. Diabetologia 39: 1263-1268

5. Brignardello E, Beltramo E, Molinatti PA et al. (1998) Dehydroepiandrosterone protects bovine retinal capillary pericytes against glucose toxicity. J Endocrinol 158: 21-26

6. Newcomb PM, Herman IM (1993) Pericyte growth and contractile phenotype: modulation by endothelial-synthesized matrix and comparison with aortic smooth muscle. J Cell Physiol 155: 385-393

7. Brownlee M (1994) Glycation and diabetic complications. Diabetes 43: 836-841

8. Thornalley PJ, Jahan I, Ng R (2001) Suppression of the accumulation of triosephosphates and increased formation of methylglyoxal in human red blood cells during hyperglycaemia by thiamine in vitro. J Biochem 129: 543-549

9. Paul RG, Bailey AJ (1999) The effect of advanced glycation end-product formation upon cell-matrix interactions. Int J Biochem Cell Biol 31: 653-660

10. Reddi AS (1985) Collagen metabolism in the retina of normal and diabetic rats. Exp Eye Res 41: 345-352 